\title{
AN ASYMPTOTIC GLRT FOR THE DETECTION OF CYCLOSTATIONARY SIGNALS
}

\author{
David Ramírez ${ }^{1}$, Louis L. Scharf ${ }^{2}$, Javier Vía ${ }^{3}$, Ignacio Santamaría ${ }^{3}$, and Peter J. Schreier ${ }^{1}$ \\ ${ }^{1}$ Signal and System Theory Group, University of Paderborn, Paderborn, Germany. \\ e-mail: \{david.ramirez,peter.schreier\}@sst.upb.de \\ ${ }^{2}$ Dept. of Mathematics, Colorado State University, Fort Collins, USA \\ e-mail: Louis.Scharf@ColoState.edu \\ ${ }^{3}$ Advanced Signal Processing Group, University of Cantabria, Santander, Spain. \\ e-mail: \{jvia,nacho\}@gtas.dicom.unican.es
}

\begin{abstract}
We derive the generalized likelihood ratio test (GLRT) for detecting cyclostationarity in scalar-valued time series. The main idea behind our approach is Gladyshev's relationship, which states that when the scalar-valued cyclostationary signal is blocked at the known cycle period it produces a vectorvalued wide-sense stationary (WSS) process. This result amounts to saying that the covariance matrix of the vector obtained by stacking all observations of the time series is block-Toeplitz if the signal is cyclostationary, and Toeplitz if the signal is wide-sense stationary. The derivation of the GLRT requires the maximum likelihood estimates of Toeplitz and block-Toeplitz matrices. This can be managed asymptotically (for large number of samples) exploiting Szegö's theorem and its generalization for vector-valued processes. Simulation results show the good performance of the proposed GLRT.
\end{abstract}

Index Terms - Cyclostationarity, generalized likelihood ratio test (GLRT), hypothesis test, maximum likelihood (ML) estimation, Toeplitz matrices.

\section{INTRODUCTION}

A zero-mean discrete-time random process $u[n]$ is called (wide-sense) cyclostationary (or periodically correlated) if there exists a positive integer $L$ such that its covariance function satisfies $\left.E\left[u[n] u^{*}[n-m]\right]=E\left[u[n+L] u^{*}[n+L-m]\right]\right]^{1}$ Cyclostationary processes model periodic phenomena occurring in science and technology, including communications, finance, acoustics, oceanography, climatology, mechanics, medicine, and biology (see [1,2] and references therein). In particular, cyclostationary signal analysis has received renewed interest in Cognitive Radio (CR) applications [3, 4]. In interweave cognitive radios, secondary users are allowed to opportunistically access the channel when the licensed

\footnotetext{
${ }^{1}$ In this paper, we only consider second-order statistics in the definition of cyclostationarity.
}

(primary) users are not present. Since digitally modulated communication signals are cyclostationary [5], this property can be exploited to detect the presence of primary users [6-8].

In this paper, we address the following hypothesis testing problem:

$$
\begin{aligned}
& \mathcal{H}_{1}: u[n] \text { is cyclostationary with period } L, \\
& \mathcal{H}_{0}: u[n] \text { is wide-sense stationary, }
\end{aligned}
$$

where the cycle period $L$ is assumed known. This problem is of wide interest, not just in the context of CR. Hence, many researchers have considered it since the original work by Gardner [9] and Hurd [10] on cyclostationary processes (see the bibliographies by [1,2]). Several noteworthy detectors of cyclostationarity have been published. In [11, 12] the authors proposed a detector exploiting the correlation that exists between a cyclostationary signal and a copy of itself shifted by the cycle frequency, which does not fully exploit the temporal structure of the process. A detector that partially exploits the temporal correlation and is capable of detecting cyclostationarity of any order is proposed in [13]. The main idea behind this detector is that the estimated cyclic correlation function must have nonzero mean when there is a cyclostationary signal present, and zero mean otherwise. This detector requires some a priori knowledge about the true cyclic correlation in order to select the best lags for the test statistic, which may not be available in many problems. On the other hand, an advantage of this detector is that it can be easily extended to vector-valued processes [6]. Finally, yet another detector is proposed in [14]. The approach followed there is similar to our approach. The key difference is that the authors of [14] propose a likelihood ratio test, i.e., the authors assume a priori knowledge of all relevant parameter values under both hypotheses, whereas we derive a generalized likelihood ratio test. That is, while [14] needs to know the second-order statistical properties of the signal to be detected, we replace the covariance functions with their maximum likelihood (ML) estimates. 


\section{PROBLEM FORMULATION}

Following an idea by Gladyshev [15], when $\mathcal{H}_{1}$ holds we can convert the scalar-valued cyclostationary time series $u[n]$ into an equivalent vector-valued wide-sense stationary (WSS) time series

$$
\mathbf{x}[n]=[u[(n-1) L+1] \quad \cdots \quad u[n L-1] \quad u[n L]]^{T} \in \mathbb{C}^{L},
$$

with matrix-valued covariance function $\mathbf{Q}[m]=E\left[\mathbf{x}[n] \mathbf{x}^{H}[n-\right.$ $m]] \in \mathbb{C}^{L \times L}$. We now stack $N$ of these vectors, $\mathbf{x}[0], \ldots, \mathbf{x}[N-$ $1]$, into the composite vector

$$
\mathbf{y}=\left[\begin{array}{llll}
\mathbf{x}^{T}[0] & \mathbf{x}^{T}[1] & \cdots & \mathbf{x}^{T}[N-1]
\end{array}\right]^{T} \in \mathbb{C}^{N L} .
$$

Since $\mathbf{x}[n]$ is WSS, it is easy to show that the covariance matrix of $\mathbf{y}$ under $\mathcal{H}_{1}$ is

$$
\mathbf{R}_{1}=\left[\begin{array}{cccc}
\mathbf{Q}[0] & \mathbf{Q}[-1] & \ldots & \mathbf{Q}[-N+1] \\
\mathbf{Q}[1] & \mathbf{Q}[0] & \ldots & \mathbf{Q}[-N+2] \\
\vdots & \vdots & \ddots & \vdots \\
\mathbf{Q}[N-1] & \mathbf{Q}[N-2] & \ldots & \mathbf{Q}[0]
\end{array}\right]
$$

which is a block-Toeplitz matrix with block size $L$. On the other hand, under $\mathcal{H}_{0}, u[n]$ is already WSS, and we may express $\mathbf{y}$ simply as

$$
\mathbf{y}=\left[\begin{array}{llll}
u[0] & u[1] & \cdots & u[N L-1]
\end{array}\right]^{T},
$$

whose covariance matrix is Toeplitz:

$$
\mathbf{R}_{0}=\left[\begin{array}{cccc}
r[0] & r[-1] & \ldots & r[-N L+1] \\
r[1] & r[0] & \ldots & r[-N L+2] \\
\vdots & \vdots & \ddots & \vdots \\
r[N L-1] & r[N L-2] & \ldots & r[0]
\end{array}\right]
$$

where $r[m]=E\left[u[n] u^{*}[n-m]\right]$ is the correlation function of $u[n]$ under $\mathcal{H}_{0}$.

Now, assuming Gaussianity, the test in (1) may be recast as the following test for the covariance structure of $\mathbf{y}$ :

$$
\begin{aligned}
& \mathcal{H}_{1}: \mathbf{y} \sim \mathcal{C} \mathcal{N}\left(\mathbf{0}, \mathbf{R}_{1}\right), \\
& \mathcal{H}_{0}: \mathbf{y} \sim \mathcal{C} \mathcal{N}\left(\mathbf{0}, \mathbf{R}_{0}\right) .
\end{aligned}
$$

We are therefore testing a Toeplitz covariance matrix against a block-Toeplitz covariance matrix with known block size.

\section{DERIVATION OF THE GLRT}

We do not assume any knowledge about the true correlation function of $u[n]$ and, therefore, our goal in this section is to derive a generalized likelihood ratio test (GLRT) for solving (2). In a GLRT, the unknown quantities are replaced by their ML estimates under their respective hypotheses, based on $M$ realizations $\mathbf{y}_{0}, \ldots, \mathbf{y}_{M-1}$ of the vector $\mathbf{y}$. The GLRT is given by

$$
\mathscr{G}=\frac{\max _{\mathbf{R}_{0}} p\left(\mathbf{y}_{0}, \ldots, \mathbf{y}_{M-1} ; \mathbf{R}_{0}\right)}{\max _{\mathbf{R}_{1}} p\left(\mathbf{y}_{0}, \ldots, \mathbf{y}_{M-1} ; \mathbf{R}_{1}\right)},
$$

where the maximization must be carried out over the sets of Toeplitz and block-Toeplitz matrices under $\mathcal{H}_{0}$ and $\mathcal{H}_{1}$, respectively. The likelihood is

$$
\begin{aligned}
& p\left(\mathbf{y}_{0}, \ldots, \mathbf{y}_{M-1}\right.\left.; \mathbf{R}_{i}\right)= \\
& \frac{1}{\pi^{L N M}\left[\operatorname{det}\left(\mathbf{R}_{i}\right)\right]^{M}} \exp \left\{-M \operatorname{tr}\left(\mathbf{R}_{i}^{-1} \hat{\mathbf{R}}\right)\right\},
\end{aligned}
$$

with the sample covariance matrix

$$
\hat{\mathbf{R}}=\frac{1}{M} \sum_{k=0}^{M-1} \mathbf{y}_{k} \mathbf{y}_{k}^{H}
$$

\subsection{ML estimates under $\mathcal{H}_{1}$}

It is well known that there is no closed-form solution for the ML estimate of Toeplitz, or block-Toeplitz, matrices [16]. Hence, as in [17] and [18], we propose to maximize the asymptotic log-likelihood (for large $N$ ) in the frequency domain. Let us first present the following theorem, which justifies this approach.

Theorem 1 As $N \rightarrow \infty$, the asymptotic log-likelihood converges in the mean square error sense to the log-likelihood:

$$
\begin{aligned}
\lim _{N \rightarrow \infty} E\left[\mid \frac{1}{N} \log p\left(\mathbf{y}_{0}, \ldots, \mathbf{y}_{M-1} ; \mathbf{R}_{1}\right)\right. & \\
\left.-\left.\log p\left(\mathbf{y}_{0}, \ldots, \mathbf{y}_{M-1} ; \mathbf{S}_{1}\left(e^{j \theta} ; N\right)\right)\right|^{2}\right] & =0,
\end{aligned}
$$

where the asymptotic log-likelihood is given by

$$
\begin{aligned}
& \log p\left(\mathbf{y}_{0}, \ldots, \mathbf{y}_{M-1} ; \mathbf{S}_{1}\left(e^{j \theta} ; N\right)\right)=-L N M \log \pi \\
& -N M \int_{-\pi}^{\pi} \log \operatorname{det}\left(\mathbf{S}_{1}\left(e^{j \theta} ; N\right)\right) \frac{d \theta}{2 \pi} \\
& -N M \int_{-\pi}^{\pi} \operatorname{tr}\left(\mathbf{S}_{1}^{-1}\left(e^{j \theta} ; N\right) \hat{\mathbf{S}}\left(e^{j \theta} ; M, N\right)\right) \frac{d \theta}{2 \pi},
\end{aligned}
$$

with $\mathbf{S}_{1}\left(e^{j \theta} ; N\right)=\mathcal{F}(\mathbf{Q}[m])$ being the Fourier transform of the matrix-valued covariance sequence $\{\mathbf{Q}[m]\}_{m=0}^{N-1}$, that is, the power spectral density matrix. The sample power spectral density matrix is

$$
\hat{\mathbf{S}}\left(e^{j \theta} ; M, N\right)=\frac{1}{M} \sum_{k=0}^{M-1} \mathbf{x}_{k}\left(e^{j \theta} ; N\right) \mathbf{x}_{k}^{H}\left(e^{j \theta} ; N\right)
$$

and $\mathbf{x}_{k}\left(e^{j \theta} ; N\right)=(1 / \sqrt{N}) \sum_{n=0}^{N-1} \mathbf{x}_{k}[n] e^{-j \theta n}$.

Proof: See [17]. 
Based on Theorem 1, we can maximize the asymptotic log-likelihood, given by (5), instead of the likelihood, given by (4). Since the power spectral density matrix $\mathbf{S}_{1}\left(e^{j \theta} ; N\right)$ has no further special structure beyond being positive definite, and the vector-valued time series does not have any particular temporal structure, its ML estimate is given by $\hat{\mathbf{S}}_{1}\left(e^{j \theta} ; N\right)=$ $\hat{\mathbf{S}}\left(e^{j \theta} ; M, N\right)$. This yields the (asymptotic) compressed loglikelihood

$$
\begin{array}{r}
\log p\left(\mathbf{y}_{0}, \ldots, \mathbf{y}_{M-1} ; \hat{\mathbf{S}}_{1}\left(e^{j \theta} ; N\right)\right)=-L N M(\log \pi+1) \\
-N M \int_{-\pi}^{\pi} \log \operatorname{det}\left(\hat{\mathbf{S}}\left(e^{j \theta} ; M, N\right)\right) \frac{d \theta}{2 \pi} .
\end{array}
$$

\subsection{ML estimates under $\mathcal{H}_{0}$}

We can also use the idea of the asymptotic log-likelihood under $\mathcal{H}_{0}$, that is, for Toeplitz matrices rather than blockToeplitz matrices. This result is known as Whittle's likelihood [19, 20], and can be seen as a particular case of (5). Concretely, the asymptotic log-likelihood is given by

$$
\begin{aligned}
& \log p\left(\mathbf{y}_{0}, \ldots, \mathbf{y}_{M-1} ; S_{0}\left(e^{j \theta} ; N\right)\right)=-L N M \log \pi \\
& -L N M \int_{-\pi}^{\pi} \log \left(S_{0}\left(e^{j \theta} ; N\right)\right) \frac{d \theta}{2 \pi} \\
& \quad-L N M \int_{-\pi}^{\pi} \frac{\hat{S}\left(e^{j \theta} ; M, N\right)}{S_{0}\left(e^{j \theta} ; N\right)} \frac{d \theta}{2 \pi},
\end{aligned}
$$

where $S_{0}\left(e^{j \theta} ; N\right)=\mathcal{F}(r[m])$ is the Fourier transform of the covariance sequence $\{r[m]\}_{m=0}^{N-1}$, the sample power spectral density is

$$
\hat{S}\left(e^{j \theta} ; M, N\right)=\frac{1}{M} \sum_{k=0}^{M-1}\left|u_{k}\left(e^{j \theta} ; N\right)\right|^{2}
$$

and

$$
u_{k}\left(e^{j \theta} ; N\right)=\frac{1}{\sqrt{L N}} \sum_{n=0}^{L N-1} u_{k}[n] e^{-j \theta n} .
$$

Again, since the scalar-valued time series under $\mathcal{H}_{0}$ does not have any particular temporal structure, it is easy to prove that the ML estimate of $S_{0}\left(e^{j \theta} ; N\right)$ is $\hat{S}_{0}\left(e^{j \theta} ; N\right)=\hat{S}\left(e^{j \theta} ; M, N\right)$, which yields the compressed log-likelihood

$$
\begin{array}{r}
\log p\left(\mathbf{y}_{0}, \ldots, \mathbf{y}_{M-1} ; \hat{S}_{0}\left(e^{j \theta} ; N\right)\right)=-L N M(\log \pi+1) \\
-L N M \int_{-\pi}^{\pi} \log \left(\hat{S}\left(e^{j \theta} ; M, N\right)\right) \frac{d \theta}{2 \pi} .
\end{array}
$$

\subsection{Asymptotic GLRT}

Based on the asymptotic log-likelihoods, the log-GLRT may be approximated as

$$
\begin{aligned}
\log \mathscr{G} \approx \log p\left(\mathbf{y}_{0}, \ldots, \mathbf{y}_{M-1} ; \hat{S}_{0}\left(e^{j \theta} ; N\right)\right) \\
-\log p\left(\mathbf{y}_{0}, \ldots, \mathbf{y}_{M-1} ; \hat{\mathbf{S}}_{1}\left(e^{j \theta} ; N\right)\right)
\end{aligned}
$$

which converges to the log-GLRT as $N \rightarrow \infty$. Now, inserting (6) and (10) into the above expression, the asymptotic logGLRT becomes 2

$$
\begin{aligned}
\log \mathscr{G} \approx \int_{-\pi}^{\pi} \log \operatorname{det}\left(\hat{\mathbf{S}}\left(e^{j \theta} ; M, N\right)\right) \frac{d \theta}{2 \pi} & \\
& -L \int_{-\pi}^{\pi} \log \left(\hat{S}\left(e^{j \theta} ; M, N\right)\right) \frac{d \theta}{2 \pi} .
\end{aligned}
$$

For finite $N$, this is an approximation of the GLRT. The advantage of this detector compared to other cyclostationarity detectors is that it naturally captures the information at all cycle frequencies.

\section{NUMERICAL RESULTS}

In this section we evaluate the performance of our detector and compare it with the detectors proposed in [11] and [13]. We consider the following scenario, which commonly appears in Cognitive Radio:

$$
\begin{aligned}
& \mathcal{H}_{1}: u[n]=(h * s)[n]+w[n], \\
& \mathcal{H}_{0}: u[n]=w[n],
\end{aligned}
$$

where $w[n]$ is additive Gaussian noise, which is wide-sense stationary with zero-mean, $s[n]$ is a communication signal and $h[n]$ is the channel impulse response. In particular, the noise has an arbitrary PSD generated by a moving average (MA) process of order 19 (at the sampling rate), the channel is Rayleigh with 10 taps (at the symbol rate) and flat power delay profile, and the transmitted signal is QPSK with rectangular shaping and a symbol rate of 300 bauds/second. Moreover, the sampling frequency is $1.2 \mathrm{kHz}$, yielding 4 samples per symbol, which coincides with the cycle period, that is, $L=4$. In our simulations we considered $N=100$ and $M=20$, so that the total number of available samples is $100 \cdot 20 \cdot 4=8000$. The coefficients of both, the channel and the MA noise model, were randomly generated in each Monte Carlo simulation, following a Gaussian distribution.

For comparison we selected the detectors proposed in [11] and [13]. For both detectors we need to select which lag(s) of the cyclic correlation function to consider. Choosing the best lag(s) optimizes performance, but generally this requires some a priori knowledge about the true cyclic correlation function, which is generally not available. For the detector [13], we thus simply considered the estimated cyclic correlation function at lags $0,1,2$ and 3. The cyclic power spectral density was estimated using a Kaiser window of length 2049. The detector [11] only takes into account a single lag. Simply choosing lag 0 would provide poor performance for this shaping, so we decided to choose the lag that maximizes the cyclic correlation function (keeping in mind that this is unrealistic in practice).

\footnotetext{
${ }^{2}$ We omit all additive and multiplicative constants that do not depend on the data.
} 


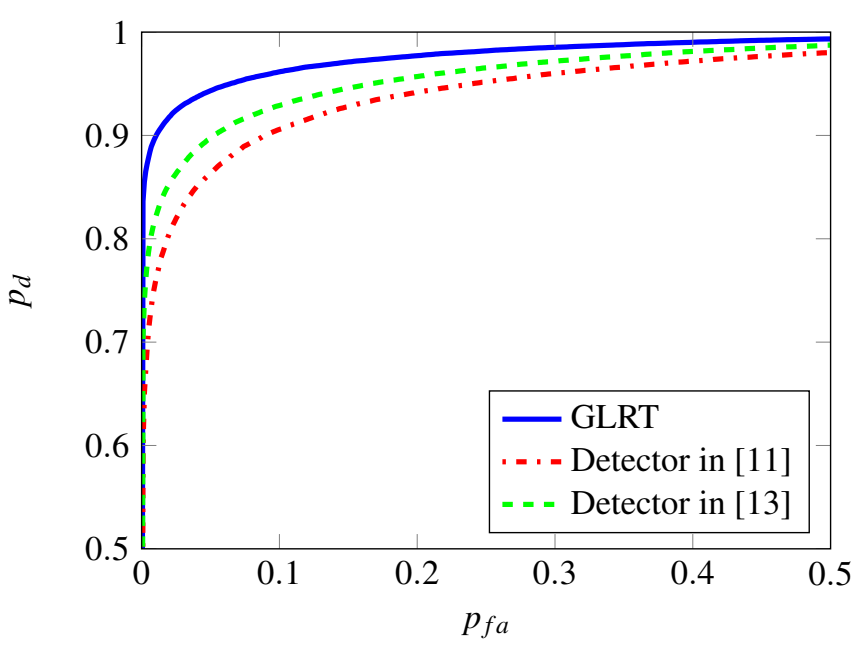

Fig. 1: ROC curve for an experiment with $L=4$ and $M=20$ snapshots of length $N=100$.

We obtained the receiver operating characteristic (ROC) curves of the three detectors for a signal-to-noise ratio (SNR) of $-10 \mathrm{~dB}$. The results, depicted in Fig. 11, show that our detector outperforms [11] and [13], even though the data is non-Gaussian. It can also be seen that detector [13] performs better than [11] for this example because the former considers the cyclic correlation function at multiple lags. Figure 2 shows the probability of missed detection vs. SNR, where similar conclusions can be drawn.

\section{CONCLUSIONS}

We have derived a detector of cyclostationarity using the relationship between a scalar-valued cyclostationary time series and a vector-valued wide-sense stationary time series. This detector is a generalized likelihood ratio test (GLRT), for which the ML estimates of a block-Toeplitz and Toeplitz matrices have to be obtained. Since there are no closed-form expressions for these ML estimates, we derived an asymptotic GLRT in the frequency domain. The main advantage of the proposed detector compared to the state-of-the-art is that it naturally exploits the information contained in all cycle frequencies. Simulation results have shown the good performance of the proposed approach.

\section{ACKNOWLEDGMENTS}

The work of L. Scharf was supported by the Airforce Office of Scientific Research under contract FA9550-10-1-0241. The work of I. Santamaría and J. Vía was supported by the Spanish Government, Ministerio de Ciencia e Innovación (MICINN), under project COSIMA (TEC2010-19545-C0403) and project COMONSENS (CSD2008-00010, CONSOLI-

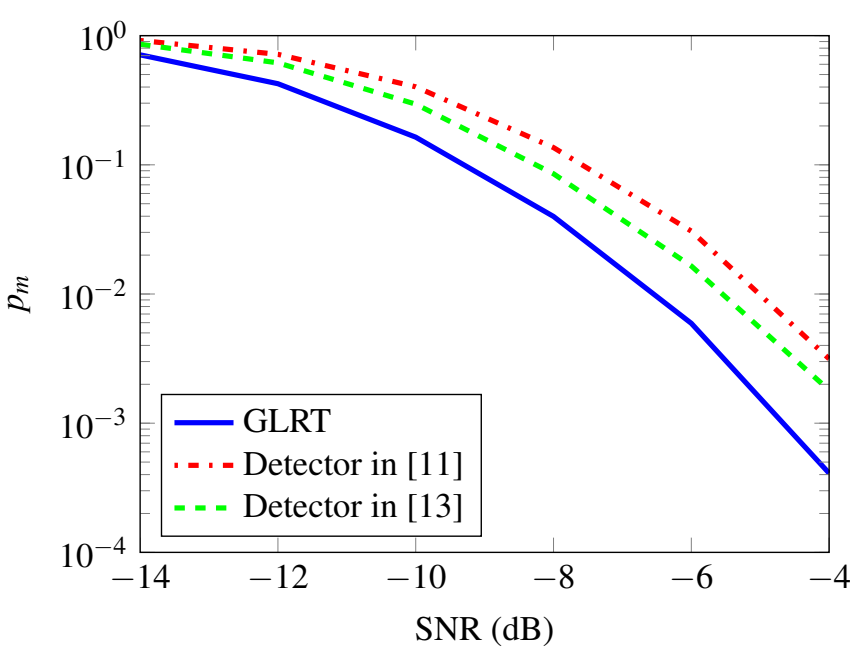

Fig. 2: Probability of missed detection for an experiment with $L=4$ and $M=20$ snapshots of length $N=100$.

DER-INGENIO 2010 Program). The work of P. Schreier was supported by the Alfried Krupp von Bohlen und Halbach Foundation, under its program "Return of German scientists from abroad."

\section{REFERENCES}

[1] W. A. Gardner, A. Napolitano, and L. Paura, "Cyclostationarity: Half a century of research," Signal Process., vol. 86, no. 4, pp. 639-697, 2006.

[2] E. Serpedin, F. Panduru, I. Sar, and G. B. Giannakis, "Bibliography on cyclostationarity," Signal Process., vol. 85, no. 12, pp. 2233-2303, 2005.

[3] J. Mitola and G. Q. Maguire Jr., "Cognitive radio: Making software radios more personal," IEEE Pers. Comm., vol. 6, pp. 13-18, Aug. 1999.

[4] J. M. Peha, "Sharing spectrum through spectrum policy reform and cognitive radio," Proc. IEEE, vol. 97, no. 4, pp. 708-719, Apr. 2009.

[5] W.A. Gardner, W. Brown, and C.-K. Chen, "Spectral correlation of modulated signals: Part ii-digital modulation," IEEE Trans. Comm., vol. 35, no. 6, pp. 595-601, 1987.

[6] J. Lundén, V. Koivunen, A. Huttunen, and H. V. Poor, "Collaborative cyclostationary spectrum sensing for cognitive radio systems," IEEE Trans. Signal Process., vol. 57, no. 11, pp. 4182-4195, Nov. 2009.

[7] J. Lunden, S. Kassam, and V. Koivunen, "Robust nonparametric cyclic correlation-based spectrum sens- 
ing for cognitive radios," IEEE Trans. Signal Process., vol. 58, no. 6, pp. 38-52, Jun. 2010.

[8] J. Renard, L. Lampe, and F. Horlin, "Spatial sign cyclicfeature detection," IEEE Trans. Signal Process., vol. 61, no. 18, pp. 4521-4531, Sept. 2013.

[9] W. A. Gardner, Introduction to Random Processes with Applications to Signal and Systems, Macmillan, New York, USA, 1985.

[10] H. L. Hurd, An investigation of periodically correlated stochastic processes, Ph.D. thesis, Duke University, 1969.

[11] S. V. Schell and W. A. Gardner, "Detection of the number of cyclostationary signals in unknown interference and noise," in Proc. Asilomar Conf. Signals, Systems and Computers, 1990.

[12] S. Enserink and D. Cochran, "On detection of cyclostationary signals," in Proc. IEEE Int. Conf. Acoust., Speech and Signal Process. (ICASSP), Detroit, USA, May 1995, pp. 2004-2007.

[13] A. V. Dandawaté and G. B. Giannakis, "Statistical tests for presence of cyclostationarity," IEEE Trans. Signal Process., vol. 42, no. 9, pp. 2355-2369, Sep. 1994.
[14] C. Andrieu and P. Duvaut, "Measure of cyclostationarity for Gaussian processes based on the likelihood ratio test," in IEEE Signal Process. Work. .Stat. Signal and Array Process., 1996, pp. 416-419.

[15] E. D. Gladyshev, "Periodically correlated random sequences," Soviet Math. Dokl., vol. 2, pp. 385-388, 1961.

[16] L. L. Scharf, Statistical Signal Processing: Detection, Estimation, and Time Series Analysis, Addison - Wesley, 1991.

[17] D. Ramírez, G. Vázquez-Vilar, R. López-Valcarce, J. Vía, and I. Santamaría, "Detection of rank- $P$ signals in cognitive radio networks with uncalibrated multiple antennas," IEEE Trans. Signal Process., vol. 59, no. 8, pp. 3764-3774, Aug. 2011.

[18] D. Ramírez, J. Vía, I. Santamaría, and L. L. Scharf, "Detection of spatially correlated Gaussian time series," IEEE Trans. Signal Process., vol. 58, no. 10, Oct. 2010.

[19] P. Whittle, "Estimation and information in time series analysis," Skand. Aktuar., vol. 35, pp. 48-60, 1952.

[20] P. Whittle, "Gaussian estimation in stationary time series," Bull. Inst. Int. Statist., vol. 39, pp. 105-129, 1962. 